\section{PTU-191 AN AUDIT OF TESTING FOR COELIAC DISEASE - ARE PATIENTS BEING INVESTIGATED APPROPRIATELY}

doi:10.1136/gutjnl-2013-304907.281

1,*F Leet, ' $S$ G J Williams, ${ }^{2 P}$ Bradley, ${ }^{3} \mathrm{~J}$ Butcher. ' Gastroenterology; ${ }^{2}$ Clinical Audit; ${ }^{3}$ Haematology, Ipswich Hospital NHS Trust, Ipswich, UK

Introduction Coeliac disease is a common condition, affecting up to 1 in 100 individuals in the UK. The average age of diagnosis is now over the age of 40 with $20 \%$ of newly diagnosed cases being over 60 . In those over 60 , the delay in diagnosis may be particularly long. The purpose of this audit was to evaluate the appropriate use of tissue transglutaminase (tTG) and duodenal biopsy in the diagnosis of celiac disease in a cohort of DGH patients analysed retrospectively.

Methods 114 consecutive patients with a reactive anti tTG $>3$ were identified from the Ipswich Hospital Pathology laboratory database between January and June 2011. Those with an anti tTG titre $>7$ have positive results in our laboratory. The cohort was divided into positive and negative groups. The duodenal biopsy (DDB) results of the positive group were documented. If the patient had not had a DDB the notes were reviewed or relevant clinician contacted. The negative group's IgA level was recorded to determine the likelihood of a false negative result.

Results Of 114 patients identified from the database with an anti $\mathrm{tTG} \geq 3,69$ were female and 45 male. There were 63 positives and 51 negatives. In the positive serology group, 48 patients were already known to have coeliac disease, 4 had a negative biopsy and 11 have not had a DDB. Of these 11 patients only 2 had been referred to gastroenterology, 3 had been given a diagnosis of coeliac disease without a DDB and 6 had not been informed of their results. In the negative serology group 19 (16 adult and 3 children) were known to have coeliac disease and were on a gluten free diet. Of the remaining 27 adult patients, only 7 (26\%) had had their IgA checked, while only 3 of 5 children had had their IgA tested. 2 of these 3 were IgA deficient. The other 2 had not been tested. None of the IgA deficient cohort had undergone a duodenal biopsy to exclude a false negative result.

Conclusion The results of this audit demonstrate inconsistent application of the national guidelines ${ }^{(1)}$ for the diagnosis of celiac disease. Of greatest concern was the cohort of adult patients who were labelled as having coeliac disease without a confirmatory biopsy. There was also a significant cohort of IgA deficient patients in the negative group who may have had celiac disease. There is clearly a need for further on-going education of all healthcare professionals regarding appropriate testing to diagnose coeliac disease to ensure appropriate treatment and prior to labelling an individual with a lifelong diagnosis

Disclosure of Interest None Declared.

\section{REFERENCE}

1. Ciclitira P J, Dewar D H, McLaughlin S D, Sanders DS (2010) The Management of Adults with Coeliac Disease, British Society of Gastroenterology

\section{PTU-192 ROLE OF THE 75SEHCAT SCAN IN EVALUATING CHRONIC DIARRHOEA}

doi:10.1136/gutjnl-2013-304907.282

1."G Diana, 'A Jawhari. 'Gastroenterology, Nottingham University Hospital, Nottingham, UK

Introduction 75Se-HCAT nuclear medicine scanning is used to diagnose Bile Salt Malabsorption (BAM) in patients with chronic diarrhoea. Previous studies suggest that $30-60 \%$ of patients with previously unexplained chronic diarrhoea have impaired bile salt absorption ${ }^{(1,2)}$. The aim of this study was to evaluate the prevalence of Bile salt malabsorption among patients with chronic diarrhoea, referred for 75SeHCAT scanning, and to evaluate the outcome of treatment with bile salt sequestrants in these patients.

Methods This was a retrospective audit of patients tested in Nottingham University Hospital in 2011. The patient was given an oral dose of $370 \mathrm{kBq}$ Selenium-75-Tauroselcholic acid, a radiolabelled bile acid analogue. The patient was scanned 7 days later to assess \%age of radioactive dose retained. Retention of $<15 \%$ was considered to be abnormal. This was further defined as mild (10-15\%), moderate $(5-10 \%)$ and severe $(<5 \%)$.

Results $\mathrm{N}=130$ patients, medican age 49 yrs, $\mathrm{F}: \mathrm{M}=1.8: 1$. The clinical indication for scanning was chronic diarrhoea in all cases (mean 6 bowel actions per day, range 1 to 18). 65 patients (50\%) had impaired salt retention. These included 11 with type I disease (Ileal resection or disease), 30 with type 2 disease (idiopathic) and 24 with type 3 disease (miscellaneous disorders eg diabetes or cholecystectomy). Interestingly only $47 \%$ of patients with previous terminal ileal resections had a positive $75 \mathrm{SeHCAT}$ scan. $47 \%$ of those patients with no predisposing factors in their history also had positive scans. $84 \%$ of cases with positive test responded to treatment with bile acid sequestrants. The response appeared to be independent from severity of BAM ( $p=0.27)$ or seveity of diarrhoea $(p=0.28)$. In one third of cases the treatment was discontinued even though clinically effective, most commonly due to poor patient tolerance to its unpleasant taste.

Conclusion The prevalence of BAM is high in patients suffering from chronic diarrhoea, both where a feasible organic precipitant is present (eg previous ileal resection), and where a functional disorder such as IBS was thought to be the likely diagnosis. BAM carries no specific symptoms or markers that can help the physician identify the condition. Bile acid sequestrants are effective, but often not well tolerated. SeHCAT scanning gives objective results and can potentially increase compliance to treatment.

\section{Disclosure of Interest None Declared.}

\section{REFERENCES}

1. S Pattini, JRF Walters. Recent advances in understanding of bile acid malabsorption. British Medical Bulletin 2009; 92:79-93.

2. Marta K. Borghede, Jacob M Schlutter, Jorgen S Agnholt, Lisbet A Christensen, Lars C Gromsen, Jens F Dahlerup. Bile acid malabsorption investigated by selenium75-homocholic acud taurin scans: Cases and treatment responses to cholestyramin in 298 patients with chronic water diarrhoea. Eur J Intern Med. 2011 Dec; 22(6):e137-40.

\section{PTU-193 A NOVEL, RATIONAL APPROACH TO TREATING PRIMARY BILE ACID DIARRHOEA: A PROOF OF CONCEPT STUDY OF THE FXR AGONIST OBETICHOLIC ACID}

doi:10.1136/gutjnl-2013-304907.283

1.* J Johnston, ${ }^{2} \mathrm{~J}$ D Nolan, ${ }^{3} \mathrm{~T}$ Dew, ${ }^{4} \mathrm{D}$ Shapiro, ${ }^{5} \mathrm{~J}$ R Walters. 'Gastroenterology, Imperial College London; ${ }^{2}$ Gastroenterology, Imperial College; ${ }^{3}$ Chemical Pathology, Kings College Hospital NHS Foundation Trust, London, UK; ${ }^{4}$ Intercept Pharmaceuticals, San Diego, United States; ${ }^{5}$ Gastroenterology, Imperial College NHS Trust, London, UK

Introduction Primary (idiopathic) bile acid diarrhoea (PBAD) is a common chronic diarrhoeal condition, affecting $\sim 1 \%$ of the population, and a large proportion of patients otherwise diagnosed with IBS-D. We showed that the ileal hormone Fibroblast Growth Factor 19 (FGF19), which decreases hepatic bile acid (BA) synthesis, is reduced in this condition, resulting in excess $B A$ production and faecal BA loss. FGF19 is secreted in response to the natural farnesoid X receptor (FXR) agonist chenodeoxycholic acid (CDCA). Obeticholic acid (OCA), 6-ethyl CDCA, is a semi-synthetic derivative with $\sim 100 \mathrm{x}$ greater FXR agonist potency. We aim to determine the FGF19, BA and clinical response to OCA in PBAD patients.

Methods After a 2-week run in period, 10 patients (7F:3M, median age 47, range 24-74) with $\mathrm{PBAD}$ (SeHCAT $7 \mathrm{~d}$ retention $<10 \%$, median $4.8 \%$ ), received oral OCA $25 \mathrm{mg}$ daily, for 2 weeks. Bile acid 
sequestrants were discontinued; loperamide was allowed as rescue therapy. Patients completed symptom diaries including stool frequency and Bristol Stool-form Scale (BSFS); a diarrhoea index ([stool frequency * mean BSFS] + loperamide use [weekly mg*3]) was calculated. Fasting serum FGF19 and total BA were measured before the first dose of OCA and after $2 \mathrm{w}$ treatment. Postprandial FGF19 and BA (6h area-under-curve, AUC) were determined after the first and last OCA dose. Data (expressed as medians) were analysed by Wilcoxon paired tests and Spearman correlation.

Results OCA increased fasting FGF19 from 133 to $237 \mathrm{pg} / \mathrm{ml}$ $(p=0.007)$ at $2 w$. Most patients had an increase $>60 \%$ in fasting FGF19 and a large OCA first dose/postprandial response. Fasting BA reduced from 1.5 to $0.9 \mu \mathrm{mol} / 1(p=0.13)$ and postprandial BA AUC was lower after the $2 \mathrm{w}$ OCA treatment (from 4.9 to $3.0 \mu \mathrm{mol} / \mathrm{l}$, $\mathrm{p}=0.02)$. Clinical improvements were found in all patients, including in stool frequency ( 23 to $14 / \mathrm{wk}, \mathrm{p}=0.02$ ), BSFS (5.15 to 4.34 , $p=0.05)$ and the diarrhoea index (113 to 76, $p=0.005)$. The reduction in BA AUC $(p=0.02)$ and the increase in fasting FGF19 $(p=0.03)$ both correlated with the reduction in stool frequency. Symptoms of abdominal pain, urgency and bloating also tended to be less on OCA treatment. OCA was well tolerated and no adverse events were reported of clinical concern.

Conclusion This study has shown for the first time that rational therapy with the FXR agonist OCA in PBAD is well tolerated and effective, stimulating serum FGF19 and reducing postprandial BA, resulting in clinical improvements in stool frequency and type. We propose larger, randomised, controlled trials of OCA. [EudraCT 2011-003777-28]

Disclosure of Interest None Declared.

\section{PTU-194 THE DIAGNOSTIC UTILITY OF COELIAC SEROLOGY IN LYMPHOCYTIC DUODENOSIS}

doi:10.1136/gutjnl-2013-304907.284

1," | Aziz, 'D M Smillie, 'D S Sanders. 'Department of Gastroenterology, Royal Hallamshire Hospital, Sheffield, UK

Introduction Lymphocytic duodenosis (LD) is defined by normal villous architecture and intraepithelial lymphocytes (IELs) $>25$ per 100 enterocytes. Such patients should not be diagnosed with coeliac disease (CD), solely by histology, as recent studies have suggested other associations with LD. Coeliac serology (tissue transglutaminase [TTG] and/or endomysial antibodies [EMA]) may play a useful role although their diagnostic value in such settings is unknown.

Aims To provide diagnostic outcomes in our expanding cohort of LD patients whilst also assessing the clinical utility of coeliac serology.

Methods Two hundred patients with LD were investigated for CD and other known associations of $\mathrm{LD}$, by means of revisiting the patient's history and recent investigations including the initial coeliac serology, followed by a combination of gluten challenge, HLA typing, repeat duodenal biopsies, and exclusion of infection/inflammatory bowel disease.

In the absence of an alternative cause, a diagnosis of CD was based on the persistence or progression of LD on a gluten containing diet, the presence of HLA DQ2 or DQ8, and a clinical response to a gluten free diet.

Results 150 female, 50 male, mean age 49, SD 16, age range 17-83

An identifiable association was found in $70 \%$ of patients - with CD (20\%), NSAIDs (17\%) and H,pylori (16\%) accounting for the majority. In $30 \%$ no cause was found, although reassuringly $2 / 3 \mathrm{rd}$ normalised their histology. The role of coeliac serology in $\mathrm{LD}$ for diagnosing $\mathrm{CD}$ is shown in table 1.

Conclusion As a single test, EMA has a greater diagnostic accuracy than TTG when assessing patients with LD.

As a combination test, only the presence of both a positive EMA and a raised TTG has a $100 \%$ predictive value for CD.
Abstract PTU-194 Table 1 The diagnostic utility of coeliac serology in lymphocytic duodenosis

\begin{tabular}{|c|c|c|c|c|}
\hline $\begin{array}{c}\text { LD coeliac serology test result } \\
(\mathrm{n} 200)\end{array}$ & PPV & NPV & Sensitivity & Specificity \\
\hline If only TTG performed & $54 \%$ & $92 \%$ & $70 \%$ & $85 \%$ \\
\hline If only EMA performed & $95 \%$ & $89 \%$ & $50 \%$ & $99 \%$ \\
\hline Both TTG and EMA normal & $6 \%$ & $44 \%$ & $20 \%$ & $16 \%$ \\
\hline TTG raised but EMA normal & $33 \%$ & $83 \%$ & $30 \%$ & $85 \%$ \\
\hline TTG normal but EMA positive & $80 \%$ & $82 \%$ & $10 \%$ & $99 \%$ \\
\hline Both TTG and EMA raised & $100 \%$ & $87 \%$ & $40 \%$ & $100 \%$ \\
\hline
\end{tabular}

Therefore, although coeliac serology is useful in $\mathrm{LD}$, most cases still require further work-up for diagnostic confirmation.

Disclosure of Interest None Declared.

\section{PTU-195 THE POPULATION PREVALENCE OF GLUTEN SENSITIVITY AND THE DIAGNOSTIC YIELD IN SECONDARY GASTROINTESTINAL CARE}

doi:10.1136/gutjnl-2013-304907.285

1."| Aziz, ${ }^{2} \mathrm{M}$ Hadjivassiliou, 'S Winfield, 'N Rugg, 'A Kelsall, 'L Newrick, 'D S Sanders. 'Gastroenterology; ${ }^{2}$ Neurology, Royal Hallamshire Hospital, Sheffield, UK

Introduction Healthcare professionals commonly encounter patients complaining of gluten sensitivity (GS) in the absence of serological and histological markers for coeliac disease (CD). This clinical entity has recently been termed non-coeliac gluten sensitivity (NCGS). The aim of this study was to determine the population prevalence of GS and to ascertain the diagnostic yield in those patients referred to secondary gastrointestinal (GI) care with gluten related symptoms.

Methods A population survey was conducted during March 2012 in Sheffield, UK, comprising basic demographic information, screening for GI conditions and enquiring for GS. We also analysed diagnostic outcomes in all patients referred by GPs to a dedicated secondary care clinic (2006-2012). The referral criteria were "GI symptoms attributed to gluten ingestion." Investigations included coeliac serology (EMA \& TTG), immunoglobulins, HLA DQ2/DQ8 typing, duodenal biopsies +/- gluten challenge if indicated. A diagnosis of CD was based on a positive coeliac serology, HLA typing and histological changes according to the Marsh classification.

Results 1002 adults completed the population based survey $(55 \%$ female, age range 16-93, mean age 39 yrs).

The prevalence of GS was 13\% (129/1002, female 80\% [P < 0.0001 ], age range $18-75$, mean age $39 \mathrm{yrs})$. The proportion of GS individuals who had seen a doctor for their symptoms was $35 / 129$ $(27 \%)$. In the absence of any known organic GI disease the prevalence of individuals fulfilling the ROME III criteria for IBS in the general population was $6 \%$, with up to $80 \%$ being female $(p<0.0001)$. Patients with IBS were more likely to report GS than non-IBS patients (43\% vs. $10 \%, \mathrm{p}<0.0001)$. GS individuals described a combination of intestinal \& extra-intestinal symptoms (Table 1). Of the GS cohort, 29\% (37/129) had tried a gluten free diet (GFD) - significant factors present in those trying a GFD include longer duration of symptoms (mean 96 vs. 54 months, $p=0.013$ ), previous doctor consultations (OR 52), diarrhoea (OR 17) and abdominal pain (OR 10.3).

In secondary GI care 156 patients with GS were investigated (85\% female, mean age $39 \mathrm{yrs}$ ). A diagnosis of CD was reached in $10 \%$ with the remaining being classified as NCGS. All patients with CD were HLA positive compared to $46 \%$ of NCGS cases. 